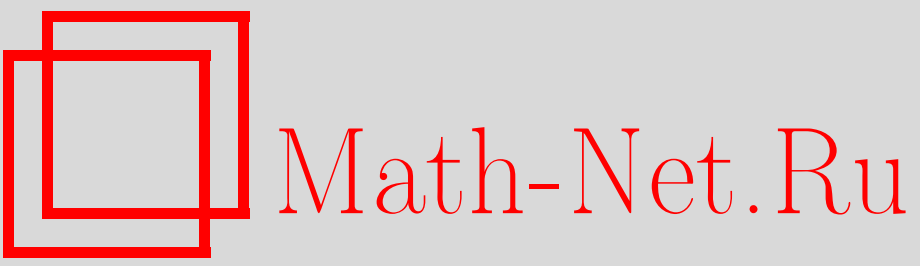

Е. Р. Аваков, А. В. Арутюнов, Теорема об обратной функции и условия экстремума для анормальных задач с незамкнутым образом, Матем. сб., 2005, том 196, номер 9, 3-22

DOI: https://doi.org/10.4213/sm1418

Использование Общероссийского математического портала Math-Net.Ru подразумевает, что вы прочитали и согласны с пользовательским соглашением

http://www. mathnet.ru/rus/agreement

Параметры загрузки:

IP : 54.157 .27 .8

26 апреля 2023 г., 07:26:14 


\author{
E.P. Аваков, А.В. Арутюнов
}

\title{
Теорема об обратной функции и условия экстремума для анормальных задач с незамкнутым образом
}

\begin{abstract}
В работе рассмотрены следующие две классические задачи: существование и оценка решения уравнения, заданного отображением $F$ в окрестности точки $x^{*}$; необходимые условия экстремума некоторой гладкой функции в точке $x^{*}$ при ограничениях типа равенства, заданных нелинейньм отображением $F$. Если образ первой производной отображения $F$ в точке $x^{*}$ не замкнут, то классические методы исследования, основанные на теоремах об обратной функции и принципе Лагранжа, не применимы. Полученные в работе результаты для указанных задач являются содержательньми для случая, когда образ первой производной отображения $F$ в точке $x^{*}$ не замкнут, и представляют собой дальнейшее развитие и обобщение классических результатов на анормальные задачи с незамкнутьм образом.

Библиограбоия: 11 названий.
\end{abstract}

\section{§1. Введение}

В статье обобщаются классическая теорема об обратной функции и принцип Лагранжа на случай, когда образ первой производной априори не предполагается замкнутым и, значит, отображение, вообще говоря, не регулярно. Для того чтобы яснее осознать смысл проведенного исследования, сформулируем доказанную в работе теорему об обратной функции параллельно с формулировкой классической теоремы.

Пусть $X, Y$ - банаховы пространства, заданы гладкое отображение $F: X \rightarrow Y$, точки $x_{*} \in X$ и $y_{*}=F\left(x_{*}\right)$. Для $y \in Y$, лежаших в окрестности $y_{*}$, рассмотрим уравнение

$$
F(x)=y
$$

относительно неизвестного $x$, лежащего в окрестности $x_{*}$. Нас будет интересовать: для всех ли указанных $y$ сушествует решение $x(y)$ уравнения $(1.1)$, которое удовлетворяет оценке через правую часть этого уравнения.

Если точка $x_{*}$ нормальна, т.е. $\operatorname{Im} F^{\prime}\left(x_{*}\right)=Y$, то по теореме об обратной функции (см. [1]) в окрестности $y_{*}$ сушествует решение $x(y)$ уравнения $(1.1)$, удовлетворяющее оценке $\left\|x(y)-x_{*}\right\| \leqslant$ const $\cdot\left\|y-y_{*}\right\|$, для которого справедливо представление

$$
x(y)=x_{*}+\left\|y-y_{*}\right\| \chi+O\left(\left\|y-y_{*}\right\|^{2}\right) .
$$

Работа выполнена при финансовой поддержке Российского фонда фундаментальных исследований (грант № 05-01-00193). 
Здесь $\chi$ - произвольное решение линейного уравнения $F^{\prime}\left(x_{*}\right) \chi=\left(y-y_{*}\right) /\left\|y-y_{*}\right\|$ при $y \neq y_{*}$, лежащее в заданном шаре с центром в точке $x_{*}$.

В настоящей работе уравнение (1.1) рассматривается для случая, когда образ $\operatorname{Im} F^{\prime}\left(x_{*}\right)$ может не быть замкнут, но при этом вьполнены ослабленные условия регулярности (они называются условиями 2-регулярности). Условие 2-регулярности $F$ по направлению $h \in X$ состоит в том, что $F^{\prime}\left(x_{*}\right) h=0, F^{\prime \prime}\left(x_{*}\right)[h, h] \in$ $\overline{\operatorname{Im} F^{\prime}\left(x_{*}\right)}$ и при этом отображение $\left(x_{1}, x_{2}\right) \rightarrow F^{\prime}\left(x_{*}\right) x_{2}+F^{\prime \prime}\left(x_{*}\right)\left[h, x_{1}\right]$ переводит $\operatorname{Ker} F^{\prime}\left(x_{*}\right) \times X$ на все $Y$. Здесь и ниже $F^{\prime \prime}\left(x_{*}\right)[\cdot, \cdot]$ - билинейное отображение, определяемое второй производной $F^{\prime \prime}\left(x_{*}\right)$. Совокупность всех описанных $h$ обозначается через $H_{0}\left(x_{*}\right)$.

В настоящей статье в предположении 2-регулярности отображения $F$ по некоторому направлению $h$ доказано сушествование решения $x(y)$ уравнения (1.1) для всех $y$, близких к $y_{*}$. Это решение удовлетворяет приводимой ниже линейно-корневой оценке. Более того, для него получено асимптотическое представление, которое, в частности, дает

$$
x(y)=x_{*}+c\left\|y-y_{*}\right\|^{1 / 2}(h+\chi)+O\left(\left\|y-y_{*}\right\|\right) .
$$

Здесь $c$ - некоторая константа, которая может быть эффективно вычислена, а $\chi-$ решение приведенного в теореме 2 линейно-квадратичного уравнения.

В случае, когда точка $x_{*}$ анормальна, т.е. $\operatorname{Im} F^{\prime}\left(x_{*}\right) \neq Y$, но образ $\operatorname{Im} F^{\prime}\left(x_{*}\right)$ замкнут, теорема об обратной функции получена в [2]. В настоящей работе результаты [2] обобщены на случай незамкнутого образа $\operatorname{Im} F^{\prime}\left(x_{*}\right)$. C их помощью получены необходимые условия для анормальных экстремальных задач с "незамкнутьм образом".

\section{§2. Теорема об обратной функции. Формулировка}

Будем предполагать, что в окрестности $x_{*}$ отображение $F$ дважды непрерьвно дифференцируемо и его вторая производная липшицева. Положим $K=\operatorname{Ker} F^{\prime}\left(x_{*}\right)$. Для произвольного фиксированного $h \in X$ определим линейное отображение

$$
G\left(x_{*}, h\right): K \times X \rightarrow Y
$$

по формуле

$$
G\left(x_{*}, h\right)\left(x_{1}, x_{2}\right)=F^{\prime}\left(x_{*}\right) x_{2}+F^{\prime \prime}\left(x_{*}\right)\left[h, x_{1}\right]
$$

Положим

$$
H\left(x_{*}\right)=\left\{h: F^{\prime}\left(x_{*}\right) h=0, F^{\prime \prime}\left(x_{*}\right)[h, h] \in \overline{\operatorname{Im} F^{\prime}\left(x_{*}\right)}\right\} .
$$

ОПРЕДЕЛЕНИЕ. Отображение $F$ называется 2-регулярным в точке $x_{*}$ по направлению $h \in H\left(x_{*}\right)$, если $G\left(x_{*}, h\right)(K \times X)=Y$. Иными словами, если

$$
\forall y \in Y \quad \exists x_{1}, x_{2}: \quad F^{\prime}\left(x_{*}\right) x_{1}=0, \quad F^{\prime}\left(x_{*}\right) x_{2}+F^{\prime \prime}\left(x_{*}\right)\left[h, x_{1}\right]=y .
$$

Множество $h \in H\left(x_{*}\right)$, по которьм $F$ 2-регулярно, обозначим через $H_{0}\left(x_{*}\right)$, т.е. положим

$$
H_{0}\left(x_{*}\right)=\left\{h \in H\left(x_{*}\right): \operatorname{Im} G\left(x_{*}, h\right)=Y\right\}
$$


Очевидно, что если точка $x_{*}$ нормальна, т.е. $\operatorname{Im} F^{\prime}\left(x_{*}\right)=Y$, то $H_{0}\left(x_{*}\right)=$ $H\left(x_{*}\right)=\operatorname{Ker} F^{\prime}\left(x_{*}\right)$. Укажем следуюшее важное свойство конуса $H_{0}\left(x_{*}\right)$. Положим

$$
\widetilde{H}_{0}\left(x_{*}\right)=\left\{h: F^{\prime}\left(x_{*}\right) h=0, F^{\prime \prime}\left(x_{*}\right)[h, h] \in \operatorname{Im} F^{\prime}\left(x_{*}\right), \operatorname{Im} G\left(x_{*}, h\right)=Y\right\} .
$$

ПРЕДЛОЖЕНИЕ 1. Конус $\widetilde{H}_{0}\left(x_{*}\right)$ всюду плотен в $H_{0}\left(x_{*}\right)$.

ДокАЗАтЕЛьство. Пусть $h \in H_{0}\left(x_{*}\right)$. Докажем сушествование сходящейся к $h$ последовательность $\left\{h_{i}\right\}$, для которой $h_{i} \in \widetilde{H}_{0}\left(x_{*}\right) \forall i$. Действительно, существуют такие последовательности $\left\{\eta_{i}\right\} \in X,\left\{y_{i}\right\} \in Y$, что $y_{i} \rightarrow 0$, $F^{\prime}\left(x_{*}\right) \eta_{i}+\frac{1}{2} F^{\prime \prime}\left(x_{*}\right)[h, h]=y_{i}$. Искомые $h_{i}$ будем искать в виде $h_{i}=h+x_{1, i}$, $x_{1, i} \in \operatorname{Ker} F^{\prime}\left(x_{*}\right)$.

Итак, рассмотрим уравнение

$$
F^{\prime}\left(x_{*}\right)\left(\eta_{i}+x_{2}\right)+\frac{1}{2} F^{\prime \prime}\left(x_{*}\right)\left[h+x_{1}\right]^{2}=0
$$

относительно неизвестных $x_{1} \in \operatorname{Ker} F^{\prime}\left(x_{*}\right), x_{2} \in X$. Проводя очевидные преобразования, приводим это уравнение к виду

$$
G\left(x_{*}, h\right)\left(x_{1}, x_{2}\right)=-y_{i}-\frac{1}{2} F^{\prime \prime}\left(x_{*}\right)\left[x_{1}\right]^{2} .
$$

Применяя к этому уравнению в точке нуль классическую теорему об обратной функции (см. [1]) (или доказанную ниже лемму 1 при $m=0$ ), получаем сушествование стремящихся к нулю решений $x_{1, i}, x_{2, i}$. Для $h_{i}=h+x_{1, i}$ по построению имеем $F^{\prime \prime}\left(x_{*}\right)\left[h_{i}\right]^{2} \in \operatorname{Im} F^{\prime}\left(x_{*}\right)$. Кроме того, при больших $i$ операторы $G\left(x_{*}, h_{i}\right)$ являются сюръективными, так как любой линейный непрерывный оператор, достаточно близкий к сюръективному, сам является сюръективньм. Таким образом, $h_{i} \in \widetilde{H}_{0}\left(x_{*}\right)$ при больших $i$. Предложение доказано.

Введем обозначение: $\theta(y)=y /\|y\|$ при $y \neq 0$ и $\theta(0)=0$. Для $y \in \operatorname{Im} F^{\prime}\left(x_{*}\right)$ положим $b(y)=\left\{\inf \|x\|: F^{\prime}\left(x_{*}\right) x=\theta(y)\right\}$. Через $B_{r}(x)$ обозначим шар радиуса $r$ с центром в точке $x$ и положим $B_{r}=B_{r}(0)$.

Теорема 1 (об обратной функции). Предположим, что отображение $F$ 2 -регулярно в точке $x_{*}$ по некоторому направлению $h \in H\left(x_{*}\right)\left(H_{0}\left(x_{*}\right) \neq \varnothing\right)$.

Тогда найдутся такие константы $r, c, c_{1}>0$, что для любого $l \in \operatorname{Im} F^{\prime}\left(x_{*}\right)$ и любого $y \in B_{r(l)}\left(y_{*}\right)$, где $r(l)=\min \left\{c r / b(l)\right.$, cr $\left.{ }^{2}\right\}$, cуществует $x(y) \in B_{r}\left(x_{*}\right)$, для которого имеют место следуюшие равенство и оченка:

$$
\begin{gathered}
F(x(y))=y \\
\left\|x(y)-x_{*}\right\| \leqslant c_{1}\left(b(l)\left\|y-y_{*}\right\|+\left\|y-y_{*}\right\|^{1 / 2}\left\|\theta\left(y-y_{*}\right)-\theta(l)\right\|^{1 / 2}\right) .
\end{gathered}
$$

Из теоремы 1 при $l=0$ вытекает следующее важное 
СлЕДСТВИЕ 1. Предположим, что $H_{0}\left(x_{*}\right) \neq \varnothing$. Тогда существуют такие константы $r, c>0$, что

$$
\forall y \in B_{r}\left(y_{*}\right) \quad \exists x(y): \quad F(x(y))=y ; \quad\left\|x(y)-x_{*}\right\| \leqslant c\left\|y-y_{*}\right\|^{1 / 2} .
$$

Теорема 2 (асимптотическое представление решения). Пусть $h \in \widetilde{H}_{0}\left(x_{*}\right)$.

Тогда существуют такие константы $r, c, c_{1}, c_{2}>0$, что для любого $l \in$ $\operatorname{Im} F^{\prime}\left(x_{*}\right)$ и любого $y \in B_{r(l)}\left(y_{*}\right)$, где $r(l)=\min \left\{c r / b(l), c r^{2}\right\}$, существует $x(y) \in B_{r}\left(x_{*}\right)$, для которого

$$
\begin{gathered}
F(x(y))=y, \\
x(y)=x_{*}+t(y)\left(h+\xi+\chi_{1}\right)+O\left(t^{2}(y)\right) .
\end{gathered}
$$

Здесь

$$
t(y)=\max \left\{\frac{c_{1}\left\|y-y_{*}\right\| b(l)}{r}, \frac{c_{2}\left\|y-y_{*}\right\|^{1 / 2}\left\|\theta\left(y-y_{*}\right)-\theta(l)\right\|^{1 / 2}}{\sqrt{r}}\right\},
$$

a $\xi, \chi_{1}, \chi_{2}-$ произвольные решения системы уравнений

$$
\begin{aligned}
F^{\prime}\left(x_{*}\right) \xi & =\frac{\left\|y-y_{*}\right\|}{t(y)} \theta(l), \\
F^{\prime}\left(x_{*}\right) \chi_{1} & =0, \\
F^{\prime}\left(x_{*}\right) \chi_{2}+F^{\prime \prime}\left(x_{*}\right)\left[h, \chi_{1}\right]=\left(\theta\left(y-y_{*}\right)-\theta(l)\right) \frac{\left\|y-y_{*}\right\|}{t^{2}(y)} & \quad-F^{\prime \prime}\left(x_{*}\right)[h, \xi]-\frac{1}{2} F^{\prime \prime}\left(x_{*}\right)\left[\xi+\chi_{1}\right]^{2},
\end{aligned}
$$

удовлетворяющие оценкам

$$
\|\xi\| \leqslant 2 b(l) \frac{\left\|y-y_{*}\right\|}{t(y)}, \quad\|\xi\| \leqslant \frac{r}{32}, \quad\left\|\chi_{1}\right\| \leqslant \frac{r}{4}, \quad\left\|\chi_{2}\right\| \leqslant \frac{r}{4} .
$$

Здесь и ниже для билинейной формы $B$ используется обозначение $B[x, x]=$ $B[x]^{2}$.

ЗАмечание 1 . Теорема 2 включает в себя, в частности, утверждение о существовании решений системы уравнений (2.5), удовлетворяющих оценкам (2.6). Отметим, что $\xi, \chi_{1}, \chi_{2}$ зависят от $y$.

ЗАмЕчАнИЕ 2. Из доказательства теоремы 2 непосредственно следует, что в ее формулировке число $b(l)$ можно заменить на произвольное $b_{0}: b_{0} \geqslant b(l)$.

ЗАмечание 3 . Если точка $x_{*}$ нормальна, то $\operatorname{Im} F^{\prime}\left(x_{*}\right)=Y, 0 \in H_{0}\left(x_{*}\right)$ и, кроме того, $\exists b_{0}: b(y) \leqslant b_{0} \forall y \in Y$. Поэтому если $x_{*}$ нормальна, то, взяв в теореме $2 h=0, l=y-y_{*}, \chi_{1}=0$, получим известную теорему об обратной функции (см. [1]), а также с учетом замечания 2 - асимптотическое представление (1.2) (при $\left.\chi=\xi c_{1} b_{0} / r\right)$. 
ЗАмечАниЕ 4. Асимптотическое представление (1.3) вытекает из теоремы 2 при $l=0, \xi=0$.

ЗАмЕчАНИЕ 5. Из приводимого ниже доказательства теоремы 2 вытекает, что участвующие в ее формулировке константы $c, c_{1}, c_{2}$ в явном виде определяются первыми двумя производными отображения $F$ в точке $x_{*}$, а также числами $\nu$ и $r_{0}$, где $\nu$ - константа Липшица отображения $F^{\prime \prime}(\cdot)$ на шаре $B_{r_{0}}\left(x_{*}\right)$. Что касается $r$, то $r$ - это произвольное число из полуинтервала $(0, \bar{r}]$, где $\bar{r}$ определяется по формуле (3.9). Сказанное относится и к теореме 1.

ЗАмечАнИЕ 6 . Если вектор $h$ не принадлежит конусу $\widetilde{H}_{0}\left(x_{*}\right)$, но $h \in H_{0}\left(x_{*}\right)$, то для него также справедливо асимптотическое представление решения (1.1), аналогичное приведенному в теореме 2 . Соответствующий результат анонсирован в [3], правда, там вследствие опечатки в формуле (7) в последних квадратных скобках перед вектором $\xi$ пропушен множитель $\left\|y-y_{*}\right\| / t(y)$.

Теорема 1 является следствием теоремы 2, однако мы сформулировали теорему 1 отдельно, так как для приложений часто достаточно бывает лишш оценки (2.2) без асимптотической формулы для решения. Доказательства теорем приводятся ниже, а здесь проиллюстрируем их на примерах. В них уравнение (1.1) исследуется в окрестности анормальной точки, и поэтому к ним классическая теорема об обратной функции (см. [1; с. 161]) не применима. В то же время предположения теорем 1 и 2 в них выполняются.

ПримеР 1. Пусть $X=\mathbb{R} \times C[0,1] \times C[0,1], Y=C[0,1], x=\left(x_{0}, x_{1}, x_{2}\right)$, где $x_{0} \in \mathbb{R}, x_{1}, x_{2} \in C[0,1]$. Определим оператор $F: X \rightarrow Y$ по формуле

$$
\begin{aligned}
F(x)(t)= & \left(t-t_{1}\right)\left(x_{0}-x_{1}(t)\right)+\left(t-t_{2}\right)\left(\int_{0}^{1} x_{1}(\tau) d \tau x_{2}(t)+\int_{0}^{1} x_{2}(\tau) d \tau x_{1}(t)\right) \\
& +\psi\left(x_{0}, x_{1}(t), x_{2}(t)\right)
\end{aligned}
$$

где $t_{1} \neq t_{2}$ - заданные точки отрезка [0,1], а $\psi$ - произвольный многочлен от трех переменных степени вьше чем 2 .

Рассмотрим уравнение (1.1). Для отображения $F$ точка $x_{*}=0$ анормальна, так как образ $\operatorname{Im} F^{\prime}\left(x_{*}\right)$ не замкнут (из-за наличия множителя $\left(t-t_{1}\right)$ ).

В то же время возьмем $h=\left(h_{0}, h_{1}, h_{2}\right)$, где $h_{0}=1, h_{1} \equiv 1, h_{2} \equiv 0$. Непосредственно проверяется, что $h \in H(0)$ и $F \quad 2$-регулярно в нуле по направлению $h^{1}$. Поэтому к рассматриваемому уравнению применимы теоремы 1 и 2 . Отметим, что в этом примере образ $F^{\prime \prime}\left(x_{*}\right)\left[h, \operatorname{Ker} F^{\prime}\left(x_{*}\right)\right]$ также не замкнут.

ПримеР 2. Пусть $X=X_{1} \times X_{2} \times X_{2}, Y=C[0,1], x=\left(x_{1}, x_{2}, x_{3}\right)$, где $x_{1} \in X_{1}=C[0,1] \cap C^{1}[2 / 3,1], x_{2}, x_{3} \in X_{2}=C[0,1] \cap C^{1}[0,1 / 3]$. Норму в $X$ определим как

$$
\|x\|=\|x\|_{C[0,1]}+\left\|\dot{x}_{1}\right\|_{C[2 / 3,1]}+\left\|\dot{x}_{2}\right\|_{C[0,1 / 3]}+\left\|\dot{x}_{3}\right\|_{C[0,1 / 3]} .
$$

\footnotetext{
${ }^{1}$ При этом использется, что для любого $v \in C[0,1]$ уравнение Фредгольма второго рода $u+\int_{0}^{1} u(\tau) d \tau=v$ имеет решение $u \in C[0,1]$.
} 
Определим оператор $F: X \rightarrow Y$ по формуле $F(x)=x_{1}+x_{2} x_{3}$.

Рассмотрим уравнение (1.1). Для отображения $F$ точка $x_{*}=0$ анормальна, так как образ $\operatorname{Im} F^{\prime}(0)$ не замкнут. Отметим, что в то же время этот образ $\operatorname{Im} F^{\prime}(0)$ всюду плотен в $Y$. Непосредственно проверяется, что отображение $F$ 2-регулярно в нуле по направлению $h \in H(0)$, где $h_{1}=h_{2}=0, h_{3} \equiv 1$. Поэтому к рассматриваемому уравнению применимы теоремы 1 и 2 . Отметим, что в этом примере образ $F^{\prime \prime}(0)\left[h, \operatorname{Ker} F^{\prime}(0)\right]$ также не замкнут.

Теорема 1 обобщает на случай незамкнутого образа $\operatorname{Im} F^{\prime}\left(x_{*}\right)$ теорему об обратной функции, доказанную в [2]. Для отображений $F$ с незамкнутым образом $\operatorname{Im} F^{\prime}\left(x_{*}\right)$ в [4], по-видимому впервые, была получена следующая теорема о накрывании: если отображение $F$ 2-регулярно в точке $x_{*}$ по некоторому направлению $h \in \widetilde{H}_{0}\left(x_{*}\right)$, то $\forall r>0 \exists \rho(r)>0: F\left(B_{r}\left(x_{*}\right)\right) \supseteq B_{\rho(r)}\left(y_{*}\right)$. В отличие от этой теоремы о накрывании из [4] теорема 2 не только утверждает сушествование решений уравнения (1.1) с указанными свойствами, но и дает асимптотическое представление этого решения. Из него, в частности, вытекают (см. теорему 1) важные линейно квадратичная оценка на решение (2.2) и корневая оценка (2.3). (В терминах накрывания корневая оценка $(2.3)$ означает, что $\rho(r) \geqslant$ const $\cdot r^{2}$ при малых $r>0$.) В предположении, что $Y$ конечномерно, другой подход к теореме об обратной функции без априорных предположений нормальности предложен в [5].

\section{§3. Доказательство теорем 1 и 2}

Начнем со следуюшей леммы. Пусть даны линейный непрерывный оператор $A: X \rightarrow Y$ и $m+1$ функций $f_{i}(x): X \rightarrow Y, i=\overline{1, m+1}$. Положим $f(x)=$ $\sum_{i=1}^{m} f_{i}(x)+f_{m+1}(x)$. Рассмотрим уравнение

$$
A x=f(x) .
$$

ЛЕмма 1. Пусть существуют такие $a, r>0$, что

$$
\begin{gathered}
\forall y \in Y \quad \exists x: \quad A x=y, \quad\|x\| \leqslant a\|y\|, \\
\left\|f_{i}\left(x^{\prime \prime}\right)-f_{i}\left(x^{\prime}\right)\right\| \leqslant(2 a(m+1))^{-1}\left\|x^{\prime \prime}-x^{\prime}\right\| \quad \forall x^{\prime}, x^{\prime \prime} \in B_{r}, \\
\left\|f_{i}(0)\right\| \leqslant r\left(a 2^{m+1}(m+1)\right)^{-1}
\end{gathered}
$$

при всех $i=\overline{1, m+1}$. Тогда

а) система уравнений

$$
\begin{gathered}
A x_{1}=f_{1}(x), \\
A x_{i}=\sum_{j=1}^{i} f_{j}\left(\sum_{s=1}^{i} x_{s}\right)-\sum_{j=1}^{i-1} f_{j}\left(\sum_{s=1}^{i-1} x_{s}\right), \quad i \geqslant 2,
\end{gathered}
$$

имеет решение $\left(x_{1}, \ldots, x_{m}\right)$, удовлетворяющее оценкам

$$
\begin{gathered}
\left\|x_{1}\right\| \leqslant 2 a\left\|f_{1}(0)\right\| \leqslant \frac{r}{2^{m}(m+1)}, \\
\left\|x_{i}\right\| \leqslant 2 a\left\|f_{i}\left(\sum_{s=1}^{i-1} x_{s}\right)\right\| \leqslant \frac{r}{2^{m}(m+1)}, \quad i=\overline{2, m} ;
\end{gathered}
$$


b) для произвольных $\left(x_{1}, \ldots, x_{m}\right)$, удовлетворяющих системе уравнений (3.4) и оченке $\left\|x_{i}\right\| \leqslant r /(2(m+1)), i=\overline{1, m}$, существует такое $x_{m+1}=x_{m+1}\left(x_{1}, \ldots, x_{m}\right) \in X$, что $x=\sum_{i=1}^{m+1} x_{i}$ является решением уравнения (3.1) и справедлива оченка

$$
\left\|x_{m+1}\right\| \leqslant 2 a\left\|f_{m+1}\left(\sum_{s=1}^{m} x_{s}\right)\right\| .
$$

ДокаЗАтЕЛьство. Построим решение $\left(x_{1}, \ldots, x_{m}\right)$ системы уравнений $(3.4)$ индукцией по $i$. Пусть вначале $i=1$. Рассмотрим отображение $F(x)=A x-f_{1}(x)$. По условию оператор $A$ накрывает с коэффициентом $a^{-1}$ (см. [6]). Следовательно, в силу (3.2) по теореме Милютина о возмущении (см. [6; теорема 1.3]) отображение $F$ накрывает на $B_{r}$ с коэффициентом $1 /(2 a)$. Поэтому $F\left(B_{\rho}\right) \supseteq F(0)+1 /(2 a) B_{\rho}$ $\forall \rho \leqslant r$. Имеем

$$
\begin{aligned}
F(0) & =-f_{1}(0) \in \frac{1}{2 a} B_{\rho} \text { при } \rho=\left\|f_{1}(0)\right\| 2 a \stackrel{(3.3)}{\leqslant} r \\
& \Longrightarrow \quad \exists x_{1}: \quad F\left(x_{1}\right)=0, \quad\left\|x_{1}\right\| \leqslant \rho=\left\|f_{1}(0)\right\| 2 a .
\end{aligned}
$$

Предположим, что уже построены произвольные первые $i, i<m$, решений $x_{1}, \ldots$, $x_{i}$ системы уравнений (3.4), удовлетворяюшие оценкам (3.5). Опишем построение $x_{i+1}$. Покажем, что

$$
\left\|x_{j}\right\| \leqslant a \gamma 2^{j} \quad \forall j \leqslant i, \quad \text { где } \gamma=r\left(a 2^{m+1}(m+1)\right)^{-1} .
$$

Для этого докажем, что

$$
\left\|x_{j}\right\| \leqslant 2 a\left(\left\|f_{j}(0)\right\|+\sum_{s=1}^{j-1}\left\|f_{j-s}(0)\right\| 2^{s-1}\right), \quad j=\overline{2, i}
$$

Действительно, в силу (3.2) и (3.5)

$$
\begin{aligned}
\left\|x_{j}\right\| & \leqslant 2 a\left(\left\|f_{j}(0)\right\|+\frac{1}{2 a} \sum_{s=1}^{j-1}\left\|x_{s}\right\|\right)=2 a\left\|f_{j}(0)\right\|+\left\|x_{j-1}\right\|+\sum_{s=1}^{j-2}\left\|x_{s}\right\| \\
& \leqslant 2 a\left(\left\|f_{j}(0)\right\|+\left\|f_{j-1}(0)\right\|\right)+2\left\|x_{j-2}\right\|+2 \sum_{s=1}^{j-3}\left\|x_{s}\right\| \leqslant \cdots
\end{aligned}
$$

Продолжая эту цепочку неравенств, получаем (3.7), откуда в силу (3.3) вытекает (3.6).

Определим отображение $F_{i}: B_{r / m+1} \rightarrow Y$ по формуле $F_{i}(x)=A x-\widetilde{f}(x)$, где

$$
\widetilde{f}(x)=\sum_{j=1}^{i+1} f_{j}\left(x_{1}+\cdots+x_{i}+x\right)-\sum_{j=1}^{i} f_{j}\left(x_{1}+\cdots+x_{i}\right)
$$


$\left(x_{1}, \ldots, x_{i}\right.$ уже фиксированы). В силу (3.2) и оценки $\left\|x_{j}\right\| \stackrel{(3.6)}{\leqslant} r /(m+1)$ функция $\tilde{f}$ удовлетворяет на $B_{r /(m+1)}$ условию Липшица с константой Липшица $1 /(2 a)$. Оператор $A$ накрьвает с коэффициентом $1 / a$. Следовательно, по теореме Милютина о возмушении отображение $F=F_{i}$ накрывает на $B_{r /(m+1)}$ с коэффициентом $1 /(2 a)$. Поэтому $F\left(B_{\rho}\right) \supseteq F(0)+1 /(2 a) B_{\rho} \forall \rho \leqslant r /(m+1)$. Но, учитывая $(3.2),(3.3),(3.6)$, имеем

$$
\begin{gathered}
F(0)=-f_{i+1}\left(x_{1}+\cdots+x_{i}\right) \Longrightarrow \\
\|F(0)\| \stackrel{(3.2)}{\leqslant}\left\|f_{i+1}(0)\right\|+\frac{1}{2 a} \sum_{s=1}^{i}\left\|x_{s}\right\| \stackrel{(3.3),(3.6)}{\leqslant} \gamma\left(1+\frac{1}{2} \sum_{s=1}^{i} 2^{s}\right) \leqslant \frac{r}{2 a(m+1)} .
\end{gathered}
$$

Следовательно,

$$
\exists x_{i+1}: \quad F\left(x_{i+1}\right)=0, \quad\left\|x_{i+1}\right\| \leqslant \rho=2 a\|F(0)\|=2 a\left\|f_{i+1}\left(x_{1}+\cdots+x_{i}\right)\right\| .
$$

Построенное $x_{i+1}$ является искомым. Индукция завершена. Неравенство

$$
2 a\left\|f_{i}\left(\sum_{s=1}^{i-1} x_{s}\right)\right\| \leqslant \frac{r}{2(m+1)}
$$

вытекает из $(3.2),(3.3)$ и (3.6); а) доказано.

Докажем b). Возьмем произвольные $x_{1}, \ldots, x_{m}$, удовлетворяющие предположениям из b). Искомое $x_{m+1}$ будем искать как решение (3.4) при $i=m+1$ относительно неизвестного $x_{m+1}$. В силу проведенных выше рассуждений $F_{m}\left(B_{\rho}\right) \supseteq$ $F_{m}(0)+1 /(2 a) B_{\rho} \forall \rho \leqslant r /(m+1)$. Кроме того,

$$
\left\|F_{m}(0)\right\| \stackrel{(3.2)}{\leqslant}\left\|f_{m+1}(0)\right\|+\frac{1}{2 a m} \sum_{s=1}^{m}\left\|x_{s}\right\| \stackrel{(3.3),(3.5)}{\leqslant} \frac{r}{2 a(m+1)}
$$

Следовательно,

$\exists x_{m+1}: \quad F_{m}\left(x_{m+1}\right)=0, \quad\left\|x_{m+1}\right\| \leqslant \rho=2 a\left\|F_{m}(0)\right\|=2 a\left\|f_{m+1}\left(x_{1}+\cdots+x_{m}\right)\right\|$.

Построенное $x_{m+1}$ является искомым. Складывая уравнения в (3.4) по всем $i \leqslant$ $m+1$ и полагая $x=\sum_{i=1}^{m+1} x_{i}$, получаем искомое решение (3.1). Лемма доказана.

ДокАЗАТЕЛЬСТво ТЕОРЕмЫ 2. Для удобства будем считать, что $\|h\| \leqslant 1$, $x_{*}=0, y_{*}=0$. Для отображения $F$ справедливо представление (это лемма Адамара):

$$
F(x)=F^{\prime}\left(x_{*}\right) x+\frac{1}{2} F^{\prime \prime}\left(x_{*}\right)[x, x]+R(x)[x, x] .
$$

Здесь при каждом фиксированном $x R(x)$ - это симметричное билинейное отображение, причем $R(0)=0$ и при некотором $r_{0}>0$ отображение $R$ удовлетворяет 
на $B_{r_{0}}$ условию Липшица с некоторой константой $\nu$. В силу 2-регулярности отображения $F$ по направлению $h$ и теоремы Банаха об открытом отображении, примененной к линейному оператору $G\left(x_{*}, h\right)$, сушествует такое $a>1$, что

$$
\forall y \in Y \quad \exists x_{1} \in K, x_{2} \in X: \quad\left\|x_{1}\right\|+\left\|x_{2}\right\| \leqslant a\|y\|, \quad G\left(x_{*}, h\right)\left(x_{1}, x_{2}\right)=y .
$$

Положим

$$
c_{3}=\max \left\{1 ; \sup \left\|F^{\prime \prime}\left(x_{*}\right)\left[x_{1}, x_{2}\right]\right\|,\left\|x_{1}\right\| \leqslant 1,\left\|x_{2}\right\| \leqslant 1\right\}, \quad \gamma_{0}=4 \nu .
$$

Возьмем произвольное $r \in(0, \bar{r}]$, где

$$
\bar{r}=\min \left\{\frac{1}{16 a c_{3}}, \frac{1}{64 a \nu}, r_{0}, 1\right\} .
$$

Непосредственным вычислением получаем оценки:

$$
\begin{aligned}
&\left\|F^{\prime \prime}\left(x_{*}\right)\left[x_{1}^{\prime}, x_{2}^{\prime}\right]-F^{\prime \prime}\left(x_{*}\right)\left[x_{1}, x_{2}\right]\right\| \leqslant \frac{1}{16 a}\left(\left\|x_{1}^{\prime}-x_{1}\right\|+\left\|x_{2}^{\prime}-x_{2}\right\|\right), \\
&\left\|R\left(x_{1}\right)\left[x_{1}, x_{1}\right]-R\left(x_{2}\right)\left[x_{2}, x_{2}\right]\right\| \leqslant \frac{1}{32 a}\left(\left\|x_{1}\right\|+\left\|x_{2}\right\|\right)\left(\left\|x_{1}-x_{2}\right\|\right), \\
&\left\|F^{\prime \prime}\left(x_{*}\right)[x, x]\right\| \leqslant \frac{r}{16 a}, \quad\|R(x)[\widetilde{x}, \widetilde{x}]\| \leqslant \gamma_{0}\|x\|
\end{aligned}
$$

для любых $x, x_{1}, x_{2}, x_{1}^{\prime}, x_{2}^{\prime} \in B_{r}, \widetilde{x} \in B_{2}$.

По условию $F^{\prime \prime}\left(x_{*}\right)[h, h] \in \operatorname{Im} F^{\prime}\left(x_{*}\right)$. Поэтому $\exists \eta \in X$ :

$$
F^{\prime}\left(x_{*}\right) \eta+\frac{1}{2} F^{\prime \prime}\left(x_{*}\right)[h, h]=0, \quad\|\eta\| \leqslant b_{1},
$$

где $b_{1}=b\left(F^{\prime \prime}\left(x_{*}\right)[h, h]\right)$. Положим

$$
\begin{gathered}
\gamma_{1}=\frac{128 a c_{3}}{r}, \quad \gamma_{2}=8\left(\frac{a}{r}\right)^{1 / 2}, \\
t_{1}=r \gamma_{3}, \quad \text { где } \gamma_{3}=\min \left\{\frac{1}{32 c_{3} b_{1} a}, \frac{1}{32 a \gamma_{0}}, \frac{1}{2}\right\}, \\
\widetilde{r}(l)=\min \left\{\frac{2 t_{1}}{\gamma_{1} b(l)}, \frac{2 t_{1}^{2}}{\gamma_{2}^{2}}\right\} .
\end{gathered}
$$

Зафиксируем произвольное $y \in B_{\widetilde{r}(l)}$ и положим

$$
t_{0}(y)=\max \left\{\frac{\|y\| b(l) \gamma_{1}}{2}, \frac{\|\theta(y)-\theta(l)\|^{1 / 2}\|y\|^{1 / 2} \gamma_{2}}{2}\right\} .
$$

Используя, что $\|\theta(l)\| \leqslant 1,\|y\| \leqslant \widetilde{r}(l)$, в силу (3.14)-(3.16) имеем

$$
\begin{aligned}
t_{0}(y) \stackrel{(3.16)}{\leqslant} \max \left\{\frac{b(l)\|y\| \gamma_{1}}{2}, \frac{\|y\|^{1 / 2} \sqrt{2} \gamma_{2}}{2}\right\} \\
\stackrel{(3.15)}{\leqslant} \max \left\{\frac{\gamma_{1} b(l)}{2} \frac{2 t_{1}}{\gamma_{1} b(l)}, \frac{\gamma_{2} \sqrt{2}}{2} \frac{\sqrt{2} t_{1}}{\gamma_{2}}\right\}=t_{1} \stackrel{(3.14)}{\leqslant} \frac{1}{2} .
\end{aligned}
$$


Выберем произвольное число $t \neq 0, t \in\left[t_{0}(y), t_{1}\right]$. По условию $l \in \operatorname{Im} F^{\prime}\left(x_{*}\right)$. Поэтому сушествует такое $\xi \in X$, что

$$
F^{\prime}\left(x_{*}\right) \xi=\frac{\|y\|}{t} \theta(l), \quad\|\xi\| \leqslant 2 b(l) \frac{\|y\|}{t} .
$$

Возьмем произвольное такое $\xi$. Из (3.16), (3.18) и определения $\gamma_{1}$ получаем

$$
\|\xi\| \leqslant \frac{4}{\gamma_{1}}=\frac{r}{32 a c_{3}} .
$$

Решение уравнения $F(x)=y$ будем искать в виде

$$
x(y, t)=t\left(h+\xi+t \eta+x_{1}+t x_{2}\right),
$$

где $x_{1} \in K, x_{2} \in X, x_{1}, x_{2} \in B_{r / 4}$. В силу выбора $t$, с учетом оценок (3.13), (3.19) формулы (3.14) и того, что $x_{1}, x_{2} \in B_{r / 4}, a \geqslant 1, c_{3} \geqslant 1, t \leqslant t_{1} \leqslant 1 / 2, t_{1} \leqslant$ $r /\left(32 a b_{1}\right)$, имеем

$$
\begin{gathered}
\left\|\xi+t \eta+x_{1}+t x_{2}\right\| \stackrel{(3.13),(3.19)}{\lessgtr} \frac{r}{32 a c_{3}}+b_{1} t+\frac{r}{2} \stackrel{(3.14)}{<} r \leqslant 1 \\
\Longrightarrow\left\|t\left(h+\xi+t \eta+x_{1}+t x_{2}\right)\right\| \leqslant 2 t \stackrel{(3.14)}{\leqslant} r .
\end{gathered}
$$

Далее, подставляя (3.20) в искомое уравнение, получаем

$$
\begin{aligned}
F( & \left.\left(h+\xi+t \eta+x_{1}+t x_{2}\right)\right)=t F^{\prime}\left(x_{*}\right) h+t F^{\prime}\left(x_{*}\right) \xi+t^{2} F^{\prime}\left(x_{*}\right) \eta+t F^{\prime}\left(x_{*}\right) x_{1} \\
& +t^{2} F^{\prime}\left(x_{*}\right) x_{2}+\frac{t^{2}}{2} F^{\prime \prime}\left(x_{*}\right)[h, h]+t^{2} F^{\prime \prime}\left(x_{*}\right)[h, \xi]+t^{3} F^{\prime \prime}\left(x_{*}\right)[h, \eta] \\
& +t^{2} F^{\prime \prime}\left(x_{*}\right)\left[h, x_{1}\right]+t^{3} F^{\prime \prime}\left(x_{*}\right)\left[h, x_{2}\right]+\frac{t^{2}}{2} F^{\prime \prime}\left(x_{*}\right)\left[\xi+x_{1}\right]^{2} \\
& +t^{3} F^{\prime \prime}\left(x_{*}\right)\left[\xi+x_{1}+\frac{t}{2}\left(\eta+x_{2}\right), \eta+x_{2}\right]+t^{2} \Delta=y
\end{aligned}
$$

Здесь $\Delta=R\left(t\left(h+\xi+t \eta+x_{1}+t x_{2}\right)\right)\left[h+\xi+t \eta+x_{1}+t x_{2}\right]^{2}$.

По построению $h, x_{1} \in \operatorname{Ker} F^{\prime}\left(x_{*}\right)$, а $\xi$ и $\eta$ удовлетворяют $(3.18),(3.13)$. Используя это и деля уравнение (3.23) на $t^{2}$, приводим его к следующему виду

$$
G\left(x_{*}, h\right) v=\sum_{i=1}^{2} f_{i}(v ; y, t) .
$$

Здесь $v=\left(x_{1}, x_{2}\right)-$ переменная, $(y, t)-$ параметр и

$$
\begin{aligned}
& f_{1}(v ; y, t)=\frac{(\theta(y)-\theta(l))\|y\|}{t^{2}}-F^{\prime \prime}\left(x_{*}\right)[h, \xi]-\frac{1}{2} F^{\prime \prime}\left(x_{*}\right)\left[\xi+x_{1}\right]^{2}, \\
& f_{2}(v ; y, t)=-F^{\prime \prime}\left(x_{*}\right)\left[t\left(h+\xi+x_{1}+\frac{t}{2}\left(\eta+x_{2}\right)\right), \eta+x_{2}\right]-\Delta .
\end{aligned}
$$


Из (3.10) в силу (3.19) для любых $x_{1}, x_{1}^{\prime} \in B_{r / 4}$ имеем

$$
\left\|\frac{1}{2} F^{\prime \prime}\left(x_{*}\right)\left[\xi+x_{1}^{\prime}\right]^{2}-\frac{1}{2} F^{\prime \prime}\left(x_{*}\right)\left[\xi+x_{1}\right]^{2}\right\| \leqslant \frac{1}{4 a}\left\|x_{1}^{\prime}-x_{1}\right\| .
$$

В силу (3.13) $\|\eta\| \leqslant b_{1}$, а в силу (3.14) $t_{1} c_{3} b_{1} \leqslant 1 /(16 a)$. Отсюда получаем

$$
\begin{aligned}
& \| F^{\prime \prime}\left(x_{*}\right)\left[t\left(h+\xi+x_{1}^{\prime}+\frac{t}{2}\left(\eta+x_{2}^{\prime}\right)\right), \eta\right] \\
& \quad-F^{\prime \prime}\left(x_{*}\right)\left[t\left(h+\xi+x_{1}+\frac{t}{2}\left(\eta+x_{2}\right)\right), \eta\right] \| \\
& \leqslant t c_{3}\left(\left\|x_{1}^{\prime}-x_{1}\right\|+\frac{t}{2}\left\|x_{2}^{\prime}-x_{2}\right\|\right)\|\eta\| \leqslant \frac{1}{16 a}\left(\left\|x_{1}^{\prime}-x_{1}^{\prime \prime}\right\|+\left\|x_{2}^{\prime}-x_{2}\right\|\right) .
\end{aligned}
$$

По аналогии с выводом оценки (3.22) получаем

$$
t\left(h+\xi+x_{1}+\frac{t}{2}\left(\eta+x_{2}\right)\right) \in B_{r} \quad \forall x_{1}, x_{2} \in B_{r / 4} .
$$

Поэтому в силу (3.10), учитывая, что $t \leqslant t_{1} \leqslant 1 / 2$, для любых $x_{1}, x_{1}^{\prime}, x_{2}, x_{2}^{\prime} \in B_{r / 4}$ имеем

$$
\begin{aligned}
\| F^{\prime \prime}\left(x_{*}\right) & {\left[t\left(h+\xi+x_{1}^{\prime}+\frac{t}{2}\left(\eta+x_{2}^{\prime}\right)\right), x_{2}^{\prime}\right] } \\
& -F^{\prime \prime}\left(x_{*}\right)\left[t\left(h+\xi+x_{1}+\frac{t}{2}\left(\eta+x_{2}\right)\right), x_{2}\right] \| \\
\leqslant & \frac{1}{16 a}\left(\left\|x_{1}^{\prime}-x_{1}\right\|+\left\|x_{2}^{\prime}-x_{2}\right\|\right) .
\end{aligned}
$$

Далее, из (3.11), (3.22), (3.21), учитывая, что $\|h\| \leqslant 1$, получаем

$$
\begin{aligned}
& \| R\left(t\left(h+\xi+t \eta+x_{1}^{\prime}+t x_{2}^{\prime}\right)\right)\left[h+\xi+t \eta+x_{1}^{\prime}+t x_{2}^{\prime}\right]^{2} \\
& \quad-R\left(t\left(h+\xi+t \eta+x_{1}+t x_{2}\right)\right)\left[h+\xi+t \eta+x_{1}+t x_{2}\right]^{2} \| \\
& \quad \leqslant \frac{1}{8 a}\left(\left\|x_{1}-x_{1}^{\prime}\right\|+\left\|x_{2}-x_{2}^{\prime}\right\|\right) .
\end{aligned}
$$

Из (3.16), (3.19), учитьвая, что $\|\xi\| \leqslant 1$, получаем

$$
\begin{aligned}
\left\|f_{1}(0 ; y, t)\right\| & \leqslant \frac{\|\theta(y)-\theta(l)\|\|y\|}{t^{2}}+\left\|F^{\prime \prime}\left(x_{*}\right)[h, \xi]\right\|+\frac{1}{2}\left\|F^{\prime \prime}\left(x_{*}\right)[\xi, \xi]\right\| \\
& \stackrel{(3.16)}{\leqslant}\left(\frac{2}{\gamma_{2}}\right)^{2}+c_{3}\|\xi\|+\frac{c_{3}}{2}\|\xi\|^{2} \leqslant \frac{r}{16 a}+2 c_{3}\|\xi\| \\
& \stackrel{(3.19)}{\leqslant} \frac{r}{16 a}+\frac{r}{16 a}=\frac{r}{8 a} .
\end{aligned}
$$


Из (3.14), (3.13), (3.19) получаем

$$
\begin{gathered}
t\left\|F^{\prime \prime}\left(x_{*}\right)\left[h+\xi+\frac{t}{2} \eta, \eta\right]\right\| \leqslant t c_{3}\left(\|h\|+\|\xi\|+\frac{t}{2}\|\eta\|\right)\|\eta\| \\
\quad \begin{array}{c}
(3.14),(3.13),(3.19) \\
\leqslant
\end{array} c_{3} 2\|\eta\| \stackrel{(3.13)}{\leqslant} 2 t c_{3} b_{1} \stackrel{(3.14)}{\leqslant} \frac{r}{16 a}
\end{gathered}
$$

Имеем также

$$
\|\Delta\| \stackrel{(3.12),(3.21),(3.22)}{\leqslant} \gamma_{0} 2 t \leqslant 2 \gamma_{0} t_{1} \stackrel{(3.14)}{\leqslant} \frac{r}{16 a} .
$$

Из полученных двух неравенств заключаем, что

$$
\left\|f_{2}(0 ; y, t)\right\| \leqslant \frac{r}{8 a} .
$$

Из (3.8), (3.25)-(3.30) в силу (3.21), (3.22) следует, что выполнены все предположения леммы 1 при $m=1, x=v=\left(x_{1}, x_{2}\right), A=G\left(x_{*}, h\right)$.

Рассмотрим операторное уравнение

$$
\begin{aligned}
& F^{\prime}\left(x_{*}\right) \chi_{2}+F^{\prime \prime}\left(x_{*}\right)\left[h, \chi_{1}\right] \\
& \quad=\left(\theta\left(y-y_{*}\right)-\theta(l)\right) \frac{\left\|y-y_{*}\right\|}{t^{2}}-F^{\prime \prime}\left(x_{*}\right)[h, \xi]-\frac{1}{2} F^{\prime \prime}\left(x_{*}\right)\left[\xi+\chi_{1}\right]^{2}
\end{aligned}
$$

относительно неизвестных $v=\left(\chi_{1}, \chi_{2}\right), \chi_{1} \in \operatorname{Ker} F^{\prime}\left(x_{*}\right), \chi_{2} \in X(y, t$ - параметры). Возьмем произвольное решение этого уравнения $v_{1}=\left(\chi_{1}(y, t), \chi_{2}(y, t)\right)$, удовлетворяюшее неравенствам

$$
\left\|\chi_{i}\right\| \leqslant\left\|v_{1}\right\| \leqslant 2 a\left\|f_{1}(0 ; y, t)\right\| \stackrel{(3.29)}{\leqslant} \frac{r}{4}, \quad i=1,2 .
$$

(Такое решение сушествует в силу леммы 1.) По лемме 1 сушествует такое $v_{2}=$ $v_{2}(y, t)=\left(v_{2,1}(y, t), v_{2,2}(y, t)\right)$, что $v=\left(v_{1}+v_{2}\right)$ является решением уравнения $(3.24)$ и имеет место оценка

$$
\left\|v_{2}(y, t)\right\| \leqslant 2 a\left\|f_{2}\left(v_{1} ; y, t\right)\right\| \stackrel{(3.22),(3.12)}{\leqslant} t \cdot \text { const. }
$$

Таким образом,

$$
x(y, t)=t\left(h+\xi+t \eta+\chi_{1}(y, t)+t \chi_{2}(y, t)+v_{2,1}(y, t)+t v_{2,2}(y, t)\right)
$$

является решением уравнения (3.24). При этом в силу (3.32)

$$
\left\|v_{2, i}(y, t)\right\| \leqslant\left\|v_{2}(y, t)\right\| \leqslant t \cdot \text { const }, \quad i=1,2 .
$$

Далее, если $y \neq 0$, то $t_{0}(y)>0$ и мы положим $t(y)=t=t_{0}(y)$. В силу доказанного выше, с учетом $(3.31),(3.33),(3.34)$ заключаем, что $x(y)=x(y, t(y))$ является искомым решением (1.1), для которого справедливо представление (2.4). При этом константы $c, c_{1}, c_{2}$ определены как $c_{1}=64 a c_{3}, c_{2}=4 a, c=\min \left\{2 \gamma_{3} / \gamma_{1}, 2 \gamma_{3}^{2} / \gamma^{2}\right\}$ (см. (3.14)) и использовано, что в силу $(3.15) r(l) \leqslant \widetilde{r}(l)$, а также $\gamma_{1} / 2=c_{1} / r$, $\gamma_{2} / 2=c_{2} / \sqrt{r}$. Теорема доказана. 
ДОКАЗАТЕЛЬСТВО ТЕОРЕМЫ 1 . В силу предложения 1 конус $\widetilde{H}_{0}\left(x_{*}\right)$ непуст. Применяя к произвольному вектору $h \in \widetilde{H}_{0}\left(x_{*}\right)$ утверждение теоремы 2 и используя очевидную оценку

$\left\|x(y)-x_{*}\right\| \stackrel{(3.22)}{\leqslant} 2 t_{0}(y) \stackrel{(3.16)}{\leqslant}$ const $\cdot\left(\left\|y-y_{*}\right\| b(l)+\left\|\left(y-y_{*}\right)-\right\| y-y_{*}\|\theta(l)\|^{1 / 2}\right)$,

получаем утверждение теоремы 1.

\section{$\S 4$. Необходимые условия экстремума для задач с незамкнутым образом}

Рассмотрим задачу минимизации с ограничением

$$
f(x) \rightarrow \min , \quad x \in X, \quad F(x)=0 .
$$

Классический метод исследования задачи (4.1) основан на принципе Лагранжа, который заключается в следующем [1], [7]. Введем функцию Лагранжа $L\left(x, \lambda_{0}, y^{*}\right)=$ $\lambda_{0} f(x)+\left\langle y^{*}, F(x)\right\rangle$, где $\lambda_{0} \geqslant 0, y^{*} \in Y^{*}$. (Значок “*” означает (в зависимости от контекста) сопряженный оператор или топологически сопряженное пространство.)

Тогда если точка $x_{*}$ является локальньм минимумом рассматриваемой задачи, подпространство $\operatorname{Im} F^{\prime}\left(x_{*}\right)$ замкнуто и функция $f$ дифференцируема в точке $x_{*}$, то справедливо

$$
\exists \lambda_{0} \geqslant 0, y^{*} \in Y^{*}: \quad \frac{\partial L}{\partial x}\left(x_{*}, \lambda_{0}, y^{*}\right)=0, \quad\left(\lambda_{0}, y^{*}\right) \neq 0 .
$$

Если при этом точка $x_{*}$ нормальна, т.е. $\operatorname{Im} F^{\prime}\left(x_{*}\right)=Y$, то $\lambda_{0}>0$ и можно положить $\lambda_{0}=1$, что дает классический принцип Лагранжа

$$
f^{\prime}\left(x_{*}\right)+F^{\prime}\left(x_{*}\right) y^{*}=0 .
$$

С другой стороны, если точка $x_{*}$, в которой $F\left(x_{*}\right)=0$, анормальна, т.е. $\operatorname{Im} F^{\prime}\left(x_{*}\right) \neq Y$, и подпространство $\operatorname{Im} F^{\prime}\left(x_{*}\right)$ замкнуто, то в силу теоремы отделимости это означает, что

$$
\exists y^{*} \in Y^{*}: \quad y^{*} \neq 0, \quad F^{\prime}\left(x_{*}\right)^{*} y^{*}=0 .
$$

Таким образом, принцип Лагранжа в любой анормальной точке $x_{*}$ ограничений задачи (4.1) в предположении замкнутости $\operatorname{Im} F^{\prime}\left(x_{*}\right)$ выполняется автоматически с $\lambda_{0}=0$ независимо от минимизируемой функции $f$ и, следовательно, никакой дополнительной содержательной информации об анормальной точке не дает.

Если же подпространство $\operatorname{Im} F^{\prime}\left(x_{*}\right)$ не замкнуто, то, как показывает следующий простой пример, принцип Лагранжа, вообще говоря, неверен даже для линейной задачи. 
ПримеР 3. Пусть $Y$ - бесконечномерное гильбертово пространство, $A: Y \rightarrow Y$ - линейный компактный симметричный положительный оператор, $b \in Y$ - произвольньй вектор, не принадлежащий $\operatorname{Im} A$. Положим $X=Y, F(x)=A x$, $f(x)=\langle b, x\rangle$. Тогда $x_{*}=0$ является решением задачи (4.1), так как $\operatorname{Ker} A=\{0\}$. Однако принцип Лагранжа (4.2) не вьполняется (так как $\left.b \notin \operatorname{Im} A^{*}, \operatorname{Ker} A^{*}=\{0\}\right)$.

В то же время для задач с линейным ограничением $F(x)=0$, очевидно, справедливо включение

$$
f^{\prime}\left(x_{*}\right) \in\left(\operatorname{Ker} F^{\prime}\left(x_{*}\right)\right)^{\perp},
$$

где значок “ $\perp$ ” означает аннулятор. Следующий пример показывает, что для нелинейных ограничений даже это - более слабое, чем классический принцип Лагранжа, включение может, вообще говоря, не выполняться.

ПримеР 4 (см. [1]). Пусть $Y, A$ и $b$ - такие же, как в примере 3 . Положим $X=\mathbb{R} \times Y, x=\left(x_{0}, x_{1}\right), f(x)=x_{0}, F(x)=A x_{1}+x_{0}^{2} b$, где $x_{0} \in \mathbb{R}, x_{1} \in Y$. Тогда в задаче $(4.1) \quad x_{*}=(0,0)$ является решением, однако $f^{\prime}\left(x_{*}\right) \notin\left(\operatorname{Ker} F^{\prime}\left(x_{*}\right)\right)^{\perp}$. Отметим, что в этом примере принцип Лагранжа (4.2) также не выполняется.

Теория необходимых условий экстремума первого и второго порядка, содержательная для анормальных задач в предположении замкнутости $\operatorname{Im} F^{\prime}\left(x_{*}\right)$, развита в [8], [9]. В предположении что $Y$ конечномерно, другой подход к необходимым условиям экстремума без априорных предположений нормальности предложен в [10]. Ниже результаты [8], [9] распространяются на случай, когда образ $\operatorname{Im} F^{\prime}\left(x_{*}\right)$ априори не предполагается замкнутым.

Для вывода необходимых условий в задаче (4.1) нам потребуется описание конуса касательных векторов $T M\left(x_{*}\right)$ ко множеству $M=\left\{x: F(x)=F\left(x_{*}\right)\right\}$ в точке $x_{*}$. Напомним, что вектор $h \in X$ называется касательным ко множеству $M$ в точке $x_{*}$, если существуют $t_{1}>0$ и отображение $r:\left[0, t_{1}\right] \rightarrow X$, для которых $x_{*}+t h+r(t) \in M \forall t \in\left[0, t_{1}\right], r(t)=o(t)$. Здесь и ниже $o-$ это отображение, для которого $o(t) / t \rightarrow 0, t \rightarrow 0$.

ТЕорема 3 (о касательном конусе). Имеет место

$$
H_{0}\left(x_{*}\right) \subseteq T M\left(x_{*}\right) \subseteq H\left(x_{*}\right)
$$

ДокАЗАТЕльство. Докажем сначала левую часть включения (4.3). Оно является следствием приведенного вьше доказательства теоремы 2.

Предположим вначале, что $h \in \widetilde{H}_{0}\left(x_{*}\right)$. Возьмем $l=0$. Тогда $\xi=0$ и $v=$ $x(y, t)$, определяемое формулой (3.33), удовлетворяет (3.24). Положив в (3.33) $y=0$, получим, что $x(t)=x(0, t)$ является решением уравнения $F(x)=0$. При этом

$$
x(t)=t h+t\left(t \eta+\chi_{1}(0, t)+t \chi_{2}(0, t)+v_{2,1}(0, t)+t v_{2,2}(0, t)\right) .
$$

В силу определения функции $f_{1}$ имеем, что $f_{1}(0,0, t) \equiv 0$, откуда в силу $(3.31)$ $\chi_{i}(0, t) \equiv 0$, а в силу (3.34) $\left\|v_{2, i}(0, t)\right\|=O(t), i=1,2$. Следовательно, $x(t)=t h+$ $O\left(t^{2}\right), t \in\left(0, t_{1}\right)$, и, значит, если $h \in \widetilde{H}_{0}\left(x_{*}\right)$, то вектор $h$ является касательньг ко множеству $M$ в точке $x_{*}$. 
Пусть теперь $h \in H_{0}\left(x_{*}\right)$, однако $h \notin \widetilde{H}_{0}\left(x_{*}\right)$. В силу предложения 1 существует сходяшаяся к $h$ последовательность $\left\{h_{i}\right\}$, для которой $h_{i} \in \widetilde{H}_{0}\left(x_{*}\right) \forall i$. В силу доказанного выше $h_{i} \in T M\left(x_{*}\right)$. Но, как известно, касательный конус замкнут и поэтому $h \in T M\left(x_{*}\right)$, так как $h_{i} \rightarrow h, i \rightarrow \infty$.

Докажем правую часть включения (4.3). Действительно, пусть $h \in T M\left(x_{*}\right)$. Тогда при малых $t>0$ имеем

$0=F\left(x_{*}+t h+o(t)\right)-F\left(x_{*}\right)=F^{\prime}\left(x_{*}\right)(t h+o(t))+\frac{1}{2} F^{\prime \prime}\left(x_{*}\right)[t h+o(t)]^{2}+o\left(t^{2}\right)$.

Деля вначале на $t>0$, а затем на $t^{2}$, при $t \rightarrow 0$ имеем $F^{\prime}\left(x_{*}\right) h=0, F^{\prime \prime}\left(x_{*}\right)[h]^{2} \in$ $\overline{\operatorname{Im} F^{\prime}\left(x_{*}\right)}$. Теорема доказана.

Если точка $x_{*}$ нормальна, то теорема 3 преврашается в “геометрическую часть" теоремы Люстерника [7], а в предположении замкнутости $\operatorname{Im} F^{\prime}\left(x_{*}\right)$ она получена в [8].

Введем в рассмотрение семейство функций, зависяших от параметра $h \in H\left(x_{*}\right)$ и переменных $\left(x, \lambda_{0}, y^{*}\right) \in X \times \mathbb{R} \times Y^{*}$ :

$$
\mathscr{L}_{h}\left(x, \lambda_{0}, y^{*}\right)=\lambda_{0} f(x)+\left\langle y^{*}, F^{\prime}(x) h\right\rangle .
$$

ТеОРема 4. Пусть $x_{*}-$ точка локального минимума в задаче (4.1). Тогда для любого $h \in H\left(x_{*}\right)$, для которого подпространство $\operatorname{Im} G\left(x_{*}, h\right)$ замкнуто, существуют неравные одновременно нулю (зависящие от $h) \lambda_{0}=\lambda_{0}(h) \geqslant 0$, $y^{*}=y^{*}(h) \in Y^{*}$, для которых выполняется

$$
\begin{gathered}
\frac{\partial \mathscr{L}_{h}}{\partial x}\left(x_{*}, \lambda_{0}, y^{*}\right) \in\left(\operatorname{Ker} F^{\prime}\left(x_{*}\right)\right)^{\perp}, \\
F^{\prime}\left(x_{*}\right)^{*} y^{*}=0 .
\end{gathered}
$$

При этом если $h \in H_{0}\left(x_{*}\right)$, то $\lambda_{0}(h)>0$.

ДокаЗАтельство. Зафиксируем произвольньй вектор $h \in H\left(x_{*}\right)$. Рассмотрим два случая. Пусть вначале $h \in H_{0}\left(x_{*}\right)$. Тогда по теореме 3 оба вектора $h$ и $(-h)$ являются касательными ко множеству $M$ в точке $x_{*}$. Отсюда легко вытекает (см., например, [10]), что

$$
\left\langle f^{\prime}\left(x_{*}\right), h\right\rangle=0
$$

Рассмотрим множество

$$
\begin{aligned}
& W(h)=\left\{(\mu, y): \text { сушествуют } x_{1} \in \operatorname{Ker} F^{\prime}\left(x_{*}\right), x_{2} \in X,\right. \\
& \text { для которых } \left.\mu>\left\langle f^{\prime}\left(x_{*}\right), x_{1}\right\rangle, y=G\left(x_{*}, h\right)\left(x_{1}, x_{2}\right)\right\} .
\end{aligned}
$$

Множество $W(h)$ вьпукло, и в силу сюръективности оператора $G\left(x_{*}, h\right)$ (ведь $\left.h \in H_{0}\left(x_{*}\right)\right)$ оно имеет непустую внутренность. Докажем, что $0 \notin W(h)$. Предположим противное, т.е. $\exists \xi_{1}, \xi_{2} \in X$ :

$$
\begin{gathered}
F^{\prime}\left(x_{*}\right) \xi_{1}=0, \quad 0>\left\langle f^{\prime}\left(x_{*}\right), \xi_{1}\right\rangle \\
0=F^{\prime}\left(x_{*}\right) \xi_{2}+2 F^{\prime \prime}\left(x_{*}\right)\left[h, \xi_{1}\right] .
\end{gathered}
$$


Определим отображение

$$
\Phi: \operatorname{Ker} F^{\prime}\left(x_{*}\right) \times X \rightarrow Y, \quad \Phi\left(x_{1}, x_{2}\right)=F^{\prime}\left(x_{*}\right) x_{2}+F^{\prime \prime}\left(x_{*}\right)\left[x_{1}, x_{1}\right] .
$$

Положим $\bar{y}=F^{\prime \prime}\left(x_{*}\right)[h, h]$. Тогда $\bar{y} \in \overline{\operatorname{Im} F^{\prime}\left(x_{*}\right)}$, так как $h \in H\left(x_{*}\right)$. Непосредственно проверяется, что $\Phi(h, 0)=\bar{y}$ и для отображения $\Phi$ точка $(h, 0)$ нормальна. Поэтому по теореме Люстерника [7] в силу (4.8) найдутся такие $t_{2}>0$ и функции $r_{i}(\cdot)$, что $r_{i}(t)=o(t), i=1,2$, и

$$
\Phi\left(h(t), t \xi_{2}+r_{2}(t)\right)=\bar{y} \in \operatorname{Im} F^{\prime}\left(x_{*}\right), \quad h(t) \in \operatorname{Ker} F^{\prime}\left(x_{*}\right) \quad \forall t \in\left[0, t_{2}\right],
$$

где $h(t)=h+t \xi_{1}+r_{1}(t)$. Следовательно, $F^{\prime \prime}[h(t)]^{2}=\bar{y}-F^{\prime}\left(x_{*}\right)\left(t \xi_{2}+r_{2}(t)\right) \in$ $\overline{\operatorname{Im} F^{\prime}\left(x_{*}\right)} \Rightarrow h(t) \in H\left(x_{*}\right)$, откуда, уменьшая $t_{2}>0$, получаем $h(t) \in H_{0}\left(x_{*}\right)$ $\forall t \in\left[0, t_{2}\right]$. Отсюда в силу (4.6) и теоремы 3 имеем $\left\langle f^{\prime}\left(x_{*}\right), \xi_{1}\right\rangle=0$, что противоречит (4.7) и, значит, доказывает, что $0 \notin W(h)$. Поэтому по теореме отделимости для вьпуклых множеств сушествуют такие неравные одновременно нулю $\lambda_{0}$, $y^{*} \in Y^{*}$, что

$$
\lambda_{0} \mu+\left\langle y^{*}, y\right\rangle \geqslant 0 \quad \forall(\mu, y) \in W(h) .
$$

Отсюда, взяв $x_{2}=0$, сразу получим (4.4), а взяв $x_{1}=0$, получим (4.5). Покажем, что $\lambda_{0} \neq 0$. Предположив противное, имеем $y^{*} \neq 0$. Выберем $\bar{y} \in Y:\left\langle y^{*}, \bar{y}\right\rangle<0$. Из определения множества $W(h)$ и сюръективности оператора $G\left(x_{*}, h\right)$ вытекает, что $\exists \bar{\mu}:(\bar{\mu}, \bar{y}) \in W(h)$, откуда в силу (4.9) и так как $\lambda_{0}=0$ заключаем, что $\left\langle y^{*}, \bar{y}\right\rangle \geqslant 0$. Полученное противоречие доказывает, что $\lambda_{0} \neq 0$ и, следовательно, $\lambda_{0}>0$.

Рассмотрим второй случай: $h \in H\left(x_{*}\right) \backslash H_{0}\left(x_{*}\right)$. В силу замкнутости подпространства $\operatorname{Im} G\left(x_{*}, h\right)$ это означает, что $\exists y^{*} \neq 0: G\left(x_{*}, h\right)^{*} y^{*}=0$, откуда получаем (4.4) при $\lambda_{0}=0$ и (4.5). Теорема доказана.

Наиболее важный и интересный для приложений случай, когда подпространство $\operatorname{Im} F^{\prime}\left(x_{*}\right)$ всюду плотно в $Y$. В этом случае из теоремы 4 вытекает, что если $H_{0}\left(x_{*}\right) \neq \varnothing$, то имеет место

$$
f^{\prime}\left(x_{*}\right) \in\left(\operatorname{Ker} F^{\prime}\left(x_{*}\right)\right)^{\perp}
$$

Действительно, возьмем $h \in H_{0}\left(x_{*}\right)$. Тогда в (4.4) можно положить $\lambda_{0}(h)=1$. В силу (4.5) $\left\langle y^{*}, y\right\rangle=0 \forall y \in \operatorname{Im} F^{\prime}\left(x_{*}\right)$, откуда $y^{*}=0$, так как $\operatorname{Im} F^{\prime}\left(x_{*}\right)$ всюду плотно в $Y$. Таким образом, из (4.4) получаем (4.10).

В то же время оказывается, что в задачах, в которых образ $\operatorname{Im} F^{\prime}\left(x_{*}\right)$ всюду плотен в $Y$ и выполнено условие 2-регулярности, справедливо более сильное, чем включение (4.10), необходимое условие локального минимума, а именно - классический принцип Лагранжа (который, как показывает пример 3, может не выполняться даже для линейных задач с всюду плотньм образом). Чтобы это доказать, нам потребуется следующее 
ПРЕДЛОЖЕНИЕ 2. Пусть $N: X \rightarrow Y$ - линейнъй непрерывный оператор u $l \in X^{*}$. Определим линейный оператор $A: X \rightarrow \mathbb{R} \times Y$ формулой $A x=$ $(\langle l, x\rangle, N x)$.

Тогда для справедливости включения $l \in \operatorname{Im} N^{*}$ необходимо и достаточно, чтобь выполнялось

$$
\overline{\operatorname{Im} A} \neq \mathbb{R} \times \overline{\operatorname{Im} N}
$$

ДоКАЗАТЕЛЬСТво. Необходимость. Предположим противное, т.е. что $\overline{\operatorname{Im} A}=$ $\mathbb{R} \times \overline{\operatorname{Im} N}$. Тогда (так как $\left.\left(1,0_{Y}\right) \in \mathbb{R} \times \overline{\operatorname{Im} N}\right)$ сушествует последовательность $\left\{x_{i}\right\}$, для которой $\left\langle l, x_{i}\right\rangle \rightarrow 1, N x_{i} \rightarrow 0, i \rightarrow \infty$. Ho $l=N^{*} y^{*} \Rightarrow\left\langle l, x_{i}\right\rangle=\left\langle y^{*}, N x_{i}\right\rangle \rightarrow 0$. Получили противоречие.

Достаточность. В силу (4.11) $\overline{\operatorname{Im} A}$ является собственным замкнутым подпространством пространства $\mathbb{R} \times \overline{\operatorname{Im} N}$. Поэтому по теореме об отделимости существуют такие неравные одновременно нулю число $\alpha$ и определенный на $\overline{\operatorname{Im} N} \subseteq Y$ линейный непрерывный функционал $y_{1}^{*}$, что

$$
\alpha a+\left\langle y_{1}^{*}, y\right\rangle=0 \quad \forall(a, y) \in \operatorname{Im} A .
$$

При этом можно взять $\alpha=1$, так как если $\alpha=0$, то $y_{1}^{*} \neq 0$ и $\left\langle y_{1}^{*}, y\right\rangle=0 \forall y \in \operatorname{Im} N$, чего не может быть.

Продолжая по теореме Хана-Банаха линейный непрерывный функционал $y_{1}^{*}$ до $y^{*} \in Y^{*}$, в силу (4.12) получаем $\langle l, x\rangle+\left\langle y^{*}, N x\right\rangle=0 \forall x \in X \Rightarrow l=-N^{*} y^{*}$. Предложение доказано.

Будем предполагать, что функция $f$ удовлетворяет тем же предположениям гладкости, что и отображение $F$.

ТеОрема 5. Пусть $x_{*}$ - точка локального минимума задачи (4.1), образ $\operatorname{Im} F^{\prime}\left(x_{*}\right)$ всюду плотен в $Y$ и существует вектор $h \in \operatorname{Ker} F^{\prime}\left(x_{*}\right)$, для которого $\operatorname{Im} G\left(x_{*}, h\right)=Y$.

Тогда существует множитель Лагранжа $y^{*} \in Y^{*}$, для которого справедлив классический принцип Лагранэса

$$
f^{\prime}\left(x_{*}\right)+F^{\prime}\left(x_{*}\right) y^{*}=0
$$

u, кроме того,

$$
\frac{\partial^{2} L}{\partial x^{2}}\left(x_{*}, 1, y^{*}\right)[h, h] \geqslant 0
$$

ДокАЗАТЕЛьство. Вначале докажем (4.13). В силу предложения 2 для этого достаточно показать, что для линейного оператора $A: X \rightarrow \mathbb{R} \times Y$, определяемого формулой $A x=\left(\left\langle f^{\prime}\left(x_{*}\right), x\right\rangle, F^{\prime}\left(x_{*}\right) x\right)$, имеет место $\overline{\operatorname{Im} A} \neq \mathbb{R} \times \overline{\operatorname{Im} F^{\prime}\left(x_{*}\right)}$.

Предположим противное, т.е. пусть имеет место

$$
\overline{\operatorname{Im} A}=\mathbb{R} \times \overline{\operatorname{Im} F^{\prime}\left(x_{*}\right)} .
$$

Поскольку $\operatorname{Im} F^{\prime}\left(x_{*}\right)$ всюду плотно в $Y$, то $H\left(x_{*}\right)=\operatorname{Ker} F^{\prime}\left(x_{*}\right) \Rightarrow H_{0}\left(x_{*}\right) \neq \varnothing$. Поэтому, как отмечалось выше, имеет место (4.10). 
Введем отображение $\Phi: X \rightarrow \mathbb{R} \times Y$ по формуле $\Phi(x)=(f(x), F(x))$. Тогда $\Phi^{\prime}\left(x_{*}\right)=$ А. Положим

$$
\mathscr{H}\left(x_{*}\right)=\left\{h \in \operatorname{Ker} A: \Phi^{\prime \prime}\left(x_{*}\right)[h, h] \in \overline{\operatorname{Im} A}\right\} .
$$

Так как $h \in \operatorname{Ker} F^{\prime}\left(x_{*}\right)$, то в силу $(4.10) h \in \operatorname{Ker} A$. Поэтому так как в силу $(4.15)$ $\overline{\operatorname{Im} A}=\mathbb{R} \times Y$, получаем, что $h \in \mathscr{H}\left(x_{*}\right)$.

Докажем, что отображение $\Phi$ 2-регулярно в точке $x_{*}$ по данному направлению $h$, т.е. $\forall a \in \mathbb{R}, y \in Y \quad \exists x_{1} \in \operatorname{Ker} A, x_{2} \in X$ :

$$
\begin{aligned}
f^{\prime}\left(x_{*}\right) x_{2}+f^{\prime \prime}\left(x_{*}\right)\left[h, x_{1}\right] & =a, \\
F^{\prime}\left(x_{*}\right) x_{2}+F^{\prime \prime}\left(x_{*}\right)\left[h, x_{1}\right] & =y .
\end{aligned}
$$

Действительно, предположим противное. Тогда так как по условию

$$
\operatorname{Im} G\left(x_{*}, h\right)=Y,
$$

то в силу следствия 4.5.11 из работы [11] подпространство $\operatorname{Im} \Phi^{\prime}\left(x_{*}\right)$ замкнуто и, следовательно, по теореме об отделимости сушествуют такие не равные одновременно нулю $\mu \geqslant 0, y^{*} \in Y^{*}$, что

$$
\begin{gathered}
\mu\left\langle f^{\prime}\left(x_{*}\right), x_{2}\right\rangle+\left\langle y^{*}, F^{\prime}\left(x_{*}\right) x_{2}\right\rangle+\mu f^{\prime \prime}\left(x_{*}\right)\left[h, x_{1}\right]+\left\langle y^{*}, F^{\prime \prime}\left(x_{*}\right)\left[h, x_{1}\right]\right\rangle=0 \\
\forall x_{1} \in \operatorname{Ker} A, \quad x_{2} \in X .
\end{gathered}
$$

Взяв в (4.16) $x_{1}=0$, имеем $\mu f^{\prime}\left(x_{*}\right)+F^{\prime}\left(x_{*}\right) y^{*}=0$. Отсюда в силу $(4.15)$ и предложения 2 (необходимость) следует, что $\mu=0$. Кроме того, из (4.10) и определения $A$ заключаем, что $\operatorname{Ker} A=\operatorname{Ker} F^{\prime}\left(x_{*}\right)$. Подставляя $\mu=0$ в (4.16), с учетом вышесказанного получаем, что $\exists y^{*} \in Y^{*}, y^{*} \neq 0$ :

$$
\left\langle y^{*}, F^{\prime}\left(x_{*}\right) x_{2}\right\rangle+\left\langle y^{*}, F^{\prime \prime}\left(x_{*}\right)\left[h, x_{1}\right]\right\rangle=0 \quad \forall x_{1} \in \operatorname{Ker} F^{\prime}\left(x^{*}\right), x_{2} \in X .
$$

Полученное равенство противоречит тому, что $\operatorname{Im} G\left(x_{*}, h\right)=Y$. Таким образом, мы показали, что отображение $\Phi$ 2-регулярно в точке $x_{*}$ по направлению $h$. Следовательно, для $\Phi$ выполнены все предположения теоремы 1 . Но тогда в силу определения отображения $\Phi$ для любой окрестности $O$ точки $x_{*}$ найдутся как угодно малые $\varepsilon>0$, при которых система уравнений

$$
f(x)=f\left(x_{*}\right)-\varepsilon, \quad F(x)=0
$$

имеет решение $x \in O$. Получили противоречие с тем, что $x_{*}-$ локальньй минимум задачи (4.1). Формула (4.13) доказана.

Докажем (4.14). В силу теоремы $3 h \in T M\left(x_{*}\right)$. Поэтому существуют $t_{1}>0$ и отображение $r$, для которых $r(t)=o(t), F\left(x_{*}+t h+r(t)\right)=0 \forall t \in\left[0, t_{1}\right]$. Поэтому так как $x_{*}$ - точка локального минимума, имеем

$$
\begin{aligned}
0 & \leqslant f\left(x_{*}+t h+r(t)\right)-f\left(x_{*}\right)=L\left(x_{*}+t h+r(t), 1, y^{*}\right)-L\left(x_{*}, 1, y^{*}\right) \\
& =\frac{\partial L}{\partial x}\left(x_{*}, 1, y^{*}\right)(t h+r(t))+\frac{1}{2} \frac{\partial^{2} L}{\partial x^{2}}\left(x_{*}, 1, y^{*}\right)[t h+r(t)]^{2}+o\left(t^{2}\right) .
\end{aligned}
$$

Деля полученное неравенство на $t^{2}$ и переходя к пределу при $t \rightarrow 0$, в силу (4.13) получаем (4.14). Теорема доказана.

Из теоремы 5 непосредственно вытекает 
СлЕДСТвИЕ. Пусть $x_{*}-$ точка локального минимума задачи (4.1), образ $\operatorname{Im} F^{\prime}\left(x_{*}\right)$ всюду плотен в $Y$ и $H_{0}\left(x_{*}\right)=H\left(x_{*}\right) \backslash\{0\}$. Тогда существует $y^{*} \in Y^{*}$, для которого выполняется классический принии Лагранжа (4.13) и, кроме того,

$$
\frac{\partial^{2} L}{\partial x^{2}}\left(x_{*}, 1, y^{*}\right)[x, x] \geqslant 0 \quad \forall x: F^{\prime}\left(x_{*}\right) x=0 .
$$

Последнее есть классическое необходимое условие второго порядка, ранее доказываемое лишь в предположении нормальности точки $x_{*}[1]$.

ЗАмечАниЕ. На самом деле доказано утверждение более сильное, чем сформулированное в теореме 5 . А именно пусть $x_{*}$ - точка локального минимума задачи (4.1) и сушествует $h \in H_{0}\left(x_{*}\right)$, для которого в утверждении теоремы 4 множитель Лагранжа $y_{2}^{*}(h)$ оказался равным нулю. Тогда справедлив классический принцип Лагранжа (4.13) и (4.14) выполняется для любого $h \in H_{0}\left(x_{*}\right)$.

Приведем пример, иллюстрируюший применение необходимых условий первого порядка из теоремы 5 .

ПрИмеР 5. Пусть $Y$ - бесконечномерное гильбертово пространство, $A: Y \rightarrow Y$ - линейный компактный симметричный положительный оператор, $b \in Y$ - произвольный вектор, не принадлежащий $\operatorname{Im} A$. Обозначим $X=\mathbb{R} \times Y \times Y$. Определим функцию $f$ на $X$ и отображение $F: X \rightarrow Y$ по формулам

$$
f(x)=\left\langle b, x_{1}\right\rangle, \quad F(x)=A x_{1}+x_{0} x_{2} .
$$

Здесь $x=\left(x_{0}, x_{1}, x_{2}\right) \in X, x_{0} \in \mathbb{R}, x_{1}, x_{2} \in Y$.

Для указанных $f, F$ рассмотрим задачу (4.1). Для нее точка $x_{*}=0$ является анормальной. При этом $\operatorname{Im} F^{\prime}(0)$ всюду плотно в $Y$, так как $\operatorname{Im} F^{\prime}(0)=\operatorname{Im} A$. Кроме того, $h=\left(1,0_{Y}, 0_{Y}\right) \in \operatorname{Ker} F^{\prime}(0)$ и, как легко видеть, $\operatorname{Im} G(0, h)=Y$.

Поэтому в точке $x_{*}=0$ применима теорема 5 . Из нее вытекает, что в задаче (4.1) точка $x_{*}=0$ локальным минимумом не является, так как непосредственно проверяется, что в этой точке классический принцип Лагранжа (4.13) не выполняется (это следует из того, что по условию $b \notin \operatorname{Im} A$ ).

В то же время в точке $x_{*}=0$ условие (4.10) вьполняется. Следовательно, вообще говоря, условие принадлежности аннулятору (4.10) слабее классического принципа Лагранжа (4.13).

\section{Список литературы}

1. Алексеев В. М., Тихомиров В. М., Фомин С. В. Оптимальное управление. М.: Наука, 1979.

2. Аваков E. Р. Теоремы об оценках в окрестности особой точки отображения // Матем. заметки. 1990. Т. 47. № 5. С. 3-13.

3. Аваков E.P., Арутюнов А.В. Об анормальных задачах с незамкнутьм образом // Докл. РАН. 2004. Т. 399. № 5. С. 583-586.

4. Sussmann H. J. High-order open mapping theorems // Directions in mathematical systems theory and optimization / ed. A. Rantzer, C.I. Byrnes. Berlin: Springer-Verlag, 2003. P. $293-316$. 
5. Арутюнов A. B. Теорема о неявной функции как реализация принципа Лагранжа. Анормалњные точки // Матем. сб. 2000. Т. 191. №1. С. 3-26.

6. Дмитрук А.В., Милютин А. А., Осмоловский Н. П. Теорема Люстерника и теория экстремума // УМН. 1980. Т. 35. №6. С. 11-46.

7. Иоффе А. Д., Тихомиров В. М. Теория экстремальных задач. М.: Наука, 1974.

8. Аваков E. Р. Условия экстремума в гладких задачах с ограничениями типа равенств // ЖВМиМФ. 1985. Т. 25. № 5. С. 680-693.

9. Аваков E.P. Необходимые условия минимума для нерегулярных задач в банаховых пространствах. Принцип максимума для анормальных задач оптимального управления // Труды МИАН. 1988. Т. 185. С. 3-29.

10. Арутюнов A. В. Условия экстремума. Анормалњные и вырожденные задачи. М.: Факториал, 1997.

11. Сухинин М. Ф. Избранные главы нелинейного анализа. М.: Изд-во РУДН, 1992.

Институт проблем управления РАН, Москва,

Российский университет дружбы народов, Москва
Поступила в редакцию 17.05 .2004 и 21.02 .2005 